\title{
Vital Signs Completion Status
}

National Cancer Institute

\section{Source}

National Cancer Institute. Vital Signs Completion Status. NCI Thesaurus. Code C83134.

A term used to describe the state or condition of the completeness of the vital signs data. 